\title{
Dissecting the Pre-Handoff Chart Biopsy: Information Seeking in the Electronic Health Record
}

\author{
Brian Hilligoss \\ School of Information, University of Michigan \\ 4341 North Quad \\ 105 S. State Street \\ Ann Arbor, Ml 48109-1285 USA \\ bhilligo@umich.edu
}

\begin{abstract}
Pre-handoff "chart biopsy" involves the brief reviewing by a clinician of a patient's record prior to accepting responsibility for the care of that patient. It is an information seeking activity enabled by electronic health records. This paper reports on a qualitative study of chart biopsies conducted by General Medicine physicians in a large quaternary teaching hospital prior to discussing possible admissions of patients from the Emergency Department (ED). The paper makes three contributions. First, this research provides an account of an information practice not previously documented and characterizes that practice as a social constructionist activity. Second, the study demonstrates that new information technologies can enable new information practices which in turn can alter the balance of participation in organizational coordination efforts. Third, the study shows that the understandings constructed from information seeking can have consequences for organizational orders.
\end{abstract}

\section{Keywords}

Electronic health record, information seeking, ethnography.

\section{INTRODUCTION}

The development of the electronic health record (EHR) has introduced new means by which clinicians may construct understandings of their patients and the care efforts of other clinicians in the service of those patients; however little is known about how physicians use EHRs. One activity that EHRs enable is an information seeking practice known informally as a "doing a chart biopsy," a targeted and typically brief examination of a patient's record prior to seeing that patient or otherwise contributing to the care of

This is the space reserved for copyright notices.

ASIST 2010, October 22-27, 2010, Pittsburgh, PA, USA.

Copyright notice continues right here. that patient. This paper provides an account of that practice, demonstrating how seeking information about a patient can be characterized as a process of socially constructing an understanding of that patient.

\section{LITERATURE REVIEW}

\section{Information Behaviors of Physicians}

Previous research has examined various information behaviors of physicians, with a heavy emphasis on information needs related to domain knowledge such as diseases, therapies and treatments (e.g., Dawes \& Sampson, 2003; Gorman \& Helfand, 1995; Green, Ciampi, \& Ellis, 2000; Savage, 1996). However, many of the day to day information needs of physicians extend beyond the formalized domain knowledge to concern informal and local issues, including specifics about particular patients (Forsythe, Buchanan, Osheroff, \& Miller, 1992; Reddy, Pratt, \& Dourish, 2002). Researchers have explored the difficulty of locating patient information in traditional paper-based records (Tang, Fafchamps, \& Shortliffe, 1994), the assembling of patient information into "bundles" to solve problems and maintain situational awareness (Gorman, et al., 2000), and the temporal aspects of seeking patient information (Reddy \& Dourish, 2002).

The sources physicians use in information seeking have been investigated in previous work. Osheroff and colleagues (1991) found that 52\% of resident's information needs could be supplied by the patient's record. One study of neo-natal intensive care unit physicians found that the patient record, including formal progress notes, was the least used source by physicians seeking patient information (Brown, Borowitz, \& Novicoff, 2004). A preference by physicians for verbal communication channels when seeking both domain knowledge and patient information has been frequently noted (Brown, et al., 2004; Covell, Uman, \& Manning, 1985; Dawes \& Sampson, 2003; Forsythe, et al., 1992; Reddy, et al., 2002). There remains a need to understand better when and how clinicians do use the patient record to satisfy information needs and how such information practices are embedded in larger work processes. 
Considerable growth in the funding and development of EHRs, coupled with mixed assessments of their abilities to positively affect safety, efficiency and quality of care (Furukawa, Raghu, \& Shao, 2010; Hillestad, et al., 2005; Sidorov, 2006) reveal a need to examine how EHRs transform or otherwise impact clinical work, including information practices. As the central organizational repositories of patient information, EHRs are poised to play important roles in meeting the information needs of physicians when those needs pertain to aspects of the patient and the care the patient has received. With respect to supporting information seeking behaviors, EHRs possess several advantages over paper-based record systems (Hersh, 1995). Of particular importance to information seeking is the fact that while paper records may be scattered across multiple locations, thereby limiting access, EHRs enable access by multiple users in different locations simultaneously. In theory, at least, a physician using an EHR may access a patient's complete documented record in one sitting, even when those records have been generated by providers in many different clinics. This affordance should have consequences for the understandings of patients that physicians develop.

\section{Patient Handoff}

One information practice that has received increased attention in recent years is the patient handoff: "the exchange between health professionals of information about a patient accompanying either a transfer of control over, or of responsibility for, the patient" (Cohen \& Hilligoss, in press). Handoffs occur when patients are moved from one facility to another or between services within a particular institution or at shift change when one team of providers ends its work day and another team takes over. These times of transition are often characterized as moments of vulnerability in the continuity of care, when quality and safety may be compromised as important information is inadequately communicated or overlooked (Behara, et al., 2005; Petersen, Brennan, O'Neil, Cook, \& Lee, 1994). For all their potential problems, handoffs may also be moments of resilience when a "fresh set of eyes" may provide a new perspective on a troubling case (Jones, et al., 2005) or may catch errors in thinking (Behara, et al., 2005; Patterson, Roth, Woods, Chow, \& Gomes, 2004).

A survey of the patient handoff literature (Cohen \& Hilligoss, 2008) reveals a heavy focus on the role of the party handing off responsibility. Less attention has been paid to the role of the receiving party in handoff, including when and how that party seeks for and uses patient information in preparation for handoff. One exception comes from Wears and colleagues (2007). Their work demonstrates how oncoming Emergency Department (ED) staff use department status boards, which include some basic patient information, to gain an overview of the proceeding shift's activities before receiving handoff. Further research is needed to explore other instances of pre- handoff gathering of patient information to shed light on handoff interactions and how they might be improved.

\section{Sensemaking and Constructionist Perspectives}

Increasingly, scholars of information-related phenomena have embraced social constructionist epistemological perspectives, producing conceptualizations of information and knowledge as socially created and contextuallyembedded, arising out of interactions rather than existing objectively, waiting to be sought and used (Dervin, 1999; Talja, 1997; Talja, Tuominen, \& Savolainen, 2005). Within organizations, the sensemaking of members and collectives impacts the processes of organizing and enacts organizational orders (Weick, 1995). While the debates regarding the philosophical assumptions of metatheory are beyond the scope of this paper, the perspective of a social constructionist epistemology is relevant here in that it naturally turns one's attention to the processes by which sense is made.

From the constructionist perspective, meaning is not discovered but constructed, and as a result, multiple competing meanings and realities may evolve. These multiple meanings in turn give rise to negotiations which establish, maintain and challenge social orders (Strauss, 1978), with consequences for the change and stability of organizations, including hospitals (Strauss, Schatzman, Ehrlich, Bucher, \& Sabshin, 1963). Information behaviors are affected by and have consequences for social order and further research is needed to connect information seeking and use with the negotiations that affect social orders.

\section{METHODS}

The analyses reported here are part of a larger, on-going constructionist Grounded Theory (Charmaz, 2006) examination of the coordination processes of physicians involved in admitting patients from the ED to the General Medicine services of a large quaternary teaching hospital. While the larger study examines handoff interactions and subsequent papers will report on how chart biopsies influence handoffs, the research question guiding the analyses reported here is: How do physicians use EHRs in preparation for patient handoff and what consequences do they perceive to result from such use?

\section{The Study Site}

This research was conducted by the author at a 900-bed quaternary teaching hospital in the Midwestern United States. The hospital admits more than 40,000 patients each year, nearly half of which are admitted through the ED. The ED has 56 resident physicians ("residents") and 41 attending physicians ("attendings"). The Internal Medicine services, including General Medicine, consist of more than 120 residents and 42 attendings. The General Medicine services include four resident services, each consisting of a team of residents supervised by an attending, and a hospitalist service, comprised entirely of attendings called "hospitalists". 
The hospital and associated health care system uses three electronic clinical information systems. "MedRec"1 is the main EHR, containing clinician notes, lab and test results, radiographic images, and other basic patient information. "EDCentral" contains records pertaining to a patient's ED stay, such as nurse triage notes, admission requests, and a "flowsheet" of time-stamped activities undertaken in the ED. "OrderCentral" is a computerized physician order entry (CPOE) system through which physicians issue orders for the care of patients. The three systems are essentially separate and have limited interconnectivity. MedRec and EDCentral are routinely accessed during chart biopsies. OrderCentral is not.

\section{Data Collection and Analysis}

Data collection involved interviews and observations. Initial participants were recruited broadly via email with cooperation from physicians with administrative responsibilities in the involved services. Following the logic of theoretical sampling (Charmaz, 2006), collection and analysis progressed iteratively, with each subsequent data collection effort aimed at locating data to fill gaps in the analysis.

Twenty-five semi-structured interviews were conducted, involving residents and attendings in the ED and General Medicine services. The author conducted over 200 hours of observations over the course of fifteen months from January 2009 through March 2010. Roughly half of this time was spent shadowing General Medicine physicians and the remainder shadowing ED physicians. More than 180 pages of typed field notes captured these observations. Access to the hospital's electronic clinical information systems permitted the researcher to examine the structure, organization and content of these systems.

Transcripts of interviews and field notes were coded using in vivo and process coding (Saldana, 2009) yielding 196 codes. One in vivo code, "doing a chart biopsy," was selected as the core category for focused coding (Charmaz, 2006). Multiple iterations of memos enabled the author to interpret and elaborate on the properties of that category.

\section{FINDINGS}

The pre-handoff chart biopsy is a relatively brief and variable information seeking activity that fits into the larger admissions process and can play a part in physicians' efforts to construct an understanding of the patient. The understandings that physicians develop from doing a chart biopsy can shape their impressions of patients and of the care provided by other clinicians and may have consequences for the handoff interaction and subsequent care of the patient. One point of clarification: of concern here are chart biopsies which occur prior to the handoff

1 Aliases have been used for the clinical information systems. conversation. This is true throughout the paper whether or not the term pre-handoff is used.

\section{Admission Process}

A brief overview of the ED to General Medicine admission process is provided here for context. Patients arrive in the ED where they are first seen by a triage nurse who takes their basic vital measurements (e.g., blood pressure, pulse, respiratory rate, temperature, etc.) and notes their "chief complaint" (e.g., the main problem or symptoms that brought the patient to the ED). These data are entered into EDCentral. The triage nurse then prioritizes the patient relative to the other patients waiting to be seen based on the nature and severity of the patient's problem. Physicians take patients in the order in which the triage nurse has prioritized them. ED physician work involves a number of activities including examining and interviewing the patients, ordering diagnostic tests and labs, and requesting consultations by surgeons and specialists. ED physicians develop a "differential diagnosis"-a list of possible diagnoses that could explain the patient's symptoms, and then attempt to "rule out" the life-threatening diagnoses. Given insurance reimbursement policies, patients cannot be kept in the ED for more than 24 hours. Other workflow pressures frequently motivate ED physicians to make a "disposition" plan in considerably less time than that. A disposition plan involves deciding whether to admit the patient for further diagnostic and treatment efforts or to discharge the patient from the hospital. If the patient is to be admitted, the disposition plan also includes determining the hospital service where the patient should be placed.

The study site is a complex organization of many medical and surgical services nearly all of which admit patients. Thus, having reached a decision to admit a patient, the ED physician must determine the most appropriate service. While hospital policies offer some guidance for placement, the variety and complexity of patient complaints and the uncertainty surrounding the diagnosis or the expected future trajectories of some patients' illnesses make it impossible for policy to clearly dictate all placement decisions. As a result physicians sometimes disagree about which service within the hospital is best situated to care for a particular patient, giving rise to the need to negotiate placement, as others have observed (e.g., Hartswood, et al., 2003; Nugus, et al., 2009). While the ED has the right to admit patients to General Medicine, General Medicine physicians may push back on particular admissions, attempting to have the patient placed on a different service or discharged rather than admitted. The pre-handoff chart biopsy, described in detail below, sometimes provides the information that General Medicine physicians use in these disposition negotiations.

Having selected a particular service to admit the patient, the ED physician then sends an alphanumeric page (i.e., admission page) to the physician overseeing admissions to that service ("admitting physician"), providing basic 
information about the admission and requesting the admitting physician call for a handoff. The information provided in the admission page varies from one admission to the next, but frequently included are: the name of the patient and the patient's EHR ID number, the name and contact information (e.g., phone number) of the individual issuing the page, and a very brief description of the patient's complaint. This page usually provides the General Medicine physician with the first notice of the impending admission. It also frequently plays a role in stimulating and guiding the pre-handoff chart biopsy.

The admission handoff between the ED physician and the General Medicine admitting physician takes place over the phone. These conversations are brief, usually lasting between five and ten minutes, although shorter and longer calls do occur. During the handoff, the ED physician "presents" the patient, frequently providing information about the patient's illness and care trajectories. The receiving physician may ask questions, including clarifying facts or requesting additional details. The call marks the transition of responsibility from the ED to the admitting service (in this case of this study, General Medicine); however since some amount of time elapses before the patient is physically moved out of the ED and onto the receiving service, there remains a "grey zone" (Apker, Mallak, \& Gibson, 2007) of shared responsibility until the transition of control is complete.

\section{The Chart Biopsy}

"Chart biopsy" is the informal term used by some clinicians (at the study site and other institutions) to label the activity of selectively examining portions of a patient's health record to gather specific data or information about that patient or to get a broader sense of the patient and the care that patient has received. The chart biopsy is an activity in which a clinician may engage multiple times while caring for a patient; however, of concern here are those chart biopsies performed by physicians prior to assuming responsibility for the care of newly admitted patients. The pre-handoff chart biopsy is conducted prior to actually seeing the patient or "taking the story" via handoff from another health care provider who has been responsible for the patient. Just as the medical procedure known as biopsy involves the targeted selection, retrieval and examination of bodily tissue to aid the process of diagnosis, so a chart biopsy involves the targeted selection of patient-related information from the health record to aid clinicians in the process of constructing an understanding of a patient.

An EHR makes a pre-handoff chart biopsy possible. Without an EHR the documents containing patient information would likely be scattered in various outpatient clinics and the ED, inaccessible to the General Medicine physician prior to taking the handoff. Drawing from their experiences at other hospitals where no EHR was in place, several participants spoke favorably about the study site's $\mathrm{EHR}$, in particular, its affordance of pre-handoff chart biopsies. In the words of one attending, handoffs at the institution where he formerly practiced would "bumble down the road" because the General Medicine physician would enter the handoff conversation without the benefit of an understanding gleaned from a chart biopsy.

Pre-handoff chart biopsies typically happen amid numerous other unrelated tasks in the busy context of a hospital where various concerns compete for the physician's time and attention. Extensive reading of a patient's record was almost never observed. Rather than gathering a complete understanding of the patient, the function of a chart biopsy is to provide an overview of the patient and, in some instances, answers to specific questions. In the words of one resident, the pre-handoff chart biopsy "just gives you kind of like an overall gist of the patient." This gist in turn helps the physician "get a very rough overview of what I'm expecting here," as another resident put it. In this respect the pre-handoff chart biopsy provides additional information beyond what may be contained in the admission page to enable the receiving physician to begin forming an impression of the patient and disposition plan.

Physicians may examine any number of different parts of the patient's record during a chart biopsy. Labs results, vital signs and radiographic images provide what some refer to as "objective data" about the patient. Provider notes contain narrative accounts and interpretations of the patient's illness, the diagnostic and therapeutic efforts undertaken, and the results of those efforts. As we shall see, what physicians look at depends in part on what questions or concerns they have about the patient and the disposition decision based on the text of the page they received.

Certain portions of the record provide salient cues for making inferences about the patient and disposition plan. For example, the provider notes table of contents page only lists the date, provider name, department, and service for each note. The contents of the note are available on a separate page via hyperlink. Scanning the table of contents, however, and simply seeing a note listed from a particular service can contribute to expectations about a patient. For example, seeing a note from a facility he recognized, one hospitalist made this predication about a patient, "She's gonna be sick: she's in the nursing home."

When constructing an understanding of a patient, physicians are often attempting to get a sense of the patient's illness trajectory. That is, physicians are trying to determine where in the progression of the illness the patient currently is. This sensemaking usually involves assembling data and information from different points in time to look for trends, as one General Medicine resident explained.

Anytime you look at a lab, like if I want to look at somebody's creatinine, that's a measure of kidney function, I can look at the one that they just got in the $\mathrm{ED}$, but then you can click on it and they can show you every test that they've had in the system, and so you can look at their trend over time. That's helpful. If 
somebody's coming in with liver disease, you can look at their liver enzymes, but then it will also show you all of their previous numbers so you can know, "is this worse than usual, same, better?' (P110)

Physicians sometimes complain that a handoff may provide a static picture, emphasizing the patient's current state, but having a sense of the trajectory of the illness and, as the quote suggests, knowing whether the patient is "worse than usual, same, better" provides a richer understanding of the patient. Similarly, physicians talk about needing to get a sense of what the patient's "baseline" is-that is, what is "normal" for this particular patient? Results that look concerning for one patient may in fact be fairly common for another patient with a long-standing chronic illness. Determining a patient's baseline and evaluating how their current condition compares is part of what some refer to as the "sick versus not sick" designation. Of course, nearly all of the patients who come through the ED have some genuine complaint, but all are not "sick" enough to warrant an admission while others are so "sick" as to need a higher level of care such as can only be provided in a critical care unit. One ED attending explained this in an interview.

And in emergency medicine a big part of what we do is, 'are you sick or are you not.' And by sick I mean big sick. I'm not talking about strep throat sick - are you really sick? Do you need to come into the hospital or not? That's a big part of our day to day activities is sorting that out. (P204)

Pre-handoff chart biopsies often involve looking in EDCentral where the patient's recent vital signs are listed. This data is useful in determining if the patient is stable enough to be cared for on a General Medicine service. EDCentral also provides the chief complaint. Finally, the flowsheet, a time-stamped list of activities undertaken in the ED, provides the receiving physician with a sense of the care trajectory undertaken in the ED. Seeing what diagnostics have or have not been ordered and what therapies have been administered can be used by the General Medicine physician in developing a sense of the thoroughness and appropriateness of ED care and the disposition plan. As the following field note excerpt illustrates, the time stamps are useful information in gathering a sense of the ED care trajectory.

P101 looks at the ED flowsheet for patient \#3 and laughs. He points out that at 13:04 the "resident sees her" and 13:46 "admitting her. He's done nothing for her. Probably hasn't even gotten labs yet." He laughs.... He notes aloud that he did not see an EKG ordered. (P101)

For all of the information available in the EHR, however, the record is certainly not complete. Rather it is an evolving account that may present a different picture of the patient and the care provided to the patient depending on when the chart biopsy is performed relative to when information is entered into the record. In the above example, an EKG had in fact been ordered, as the physician later learned during the handoff, but no entry had yet been made in the flowsheet.

Seeing what actions were taken in the ED, however, is only part of the information General Medicine physicians often need to construct full-fledged understandings of patients and care trajectories. Physicians also frequently need to know why those actions were taken. One ED attending explains that the ED physician's admission note, which contains this information, is transcribed and frequently not available prior to the handoff conversation.

The problem is - is our charts are dictated. So, they're not usually dictated by the time you have the conversation. [...] There's no thought process. You know, you can't see why-why did we do this? Or what did we consider, or rule out and whatever. (P201)

These accounts demonstrate the possibility that pre-handoff chart biopsies may contribute to inaccurate or incomplete understandings of the patients and disposition plans since EHRs provide evolving accounts of patients and care activities.

\section{Questions and Concerns Drive the Chart Biopsy}

While pre-handoff chart biopsies do provide an overall gist of the patient, they are not aimless browsing sessions. The demands of other work tasks and the pressures of time constraints make casual browsing impractical. Rather, chart biopsies are frequently driven by questions that emerge from generic overarching concerns about disposition plans and specific concerns about the patient arising from the description provided in the admission page.

Generic overarching concerns. Generic overarching concerns about disposition plans address two issues: 1) the appropriateness of admitting as opposed to discharging the patient, and 2) the appropriateness of placing the patient on the particular service. The disposition plan devised by ED staff is not automatically accepted as appropriate by General Medicine physicians. Instead, General Medicine physicians frequently use the chart biopsy as a means to evaluate the appropriateness of the plan.

First, physicians sometimes differ in their opinions regarding whether or not a particular patient's case warrants an admission. Established guidelines offer answers for certain conditions, but there are many symptoms and complaints that are as yet beyond the reach of clear protocols. Some ED and General Medicine physicians in this study acknowledged that ED staff are not always aware of what treatments and diagnostics are readily available in outpatient settings. As the following quote demonstrates, General Medicine physicians may conduct a chart biopsy to assess the decision to admit and then use the information they gather to argue for an alternative disposition plan during the subsequent handoff. 
When the ER calls up that they want to admit somebody, we'll review the record [...] and I will look at their outpatient provider notes to see if it's a chronic issue or to see if it could be otherwise managed. And you call back, and you talk to the ER physician, and sometimes they just haven't thought of another option. And you can say, "Well, you could do this or this and that would - and it doesn't look like they necessarily meet inpatient criteria, but blah, blah, blah," and you know they'll reconsider it. (P106)

As mentioned, handoffs can be moments of resilience when the party receiving responsibility brings a fresh perspective to the patient's case (Behara, et al., 2005). As the quote above demonstrates, a pre-handoff chart biopsy can play an important role in preparing the receiving party to effectively bring this fresh perspective.

Second, even when there is little question that the patient needs to be admitted to the hospital, General Medicine physicians may question the appropriateness of the placement decision. This concern involves two parts, as one hospitalist explained: "The first is, should it come to a medicine service, and if yes, should it come to our service specifically." The first part arises from the subdivision of the hospital into medical services and surgical services. Disagreements can arise over whether a patient belongs on a medical or surgical service. The second part arises from the subdivisions within Internal Medicine, which, in addition to General Medicine, includes a number of subspecialty services with admitting privileges. Among these are Cardiovascular Medicine (Cardiology), Pulmonary, Hematology and Oncology, and Gastroenterology, as well as critical care services. Given the complexity and co-morbidities of many patients admitted at the study site hospital, arguments can often be made that a given patient belongs on more than one Internal Medicine service. Knowing that these disagreements arise during handoffs, General Medicine physicians often use the chart biopsy to gather information to evaluate the placement decision before taking the handoff.

While the appropriateness of some placement decisions is debatable given the challenge of matching complex patients to "the right" service within a highly specialized and subdivided organizational structure, other placement decisions are clearly spelled out in hospital policy. Still, the patient details that indicate a particular policy applies to a given placement are sometimes overlooked in the ED. Thus, the generic overarching concerns that guide many chart biopsies include looking for evidence whether or not particular placement policies apply to the present case. For example, hospital policy also states that patients who have a Family Practice physician should be admitted to that service, but General Medicine physicians complained that ED staff frequently miss this designation. Consequently, checking whether or not a patient belongs to Family Medicine was one of the most common tasks observed during pre-handoff chart biopsies. In some cases, it was the only detail physicians checked during the chart biopsy. One resident explained why this is important:

...because otherwise you admit them and you give [the patient] to [Family Practice] the next day, and it just doesn't make sense. It should be in the same hand [sic] from the first minute. It's mainly to prevent extra work and to ensure patient continuity, patient safety, that's, for me, the main reason what I'm looking for rather than actual diagnosis or anything. (P109)

The resident, like others who participated in this study, describes the act of checking such details before taking the handoff in terms of efficiency and quality of care and safety. In this sense, the pre-handoff chart biopsy is positioned as a means of influencing organizational workflows and as a resource for enabling organizational resilience.

Patient-specific concerns. Frequently the description of the patient provided in the admission page gives rise to certain specific questions which in turn direct the information seeking during the chart biopsy. A field note example from an observation of a hospitalist provides an illustration.

He reads part of the page aloud: "'history of lung cancer. Got chemo and radiation. Here for further treatment. Worsening dyspnea.' So, why is she coming to us?" He reviews the patient's record and mumbles, "This sounds-not good." (P104)

The hospitalist in this example is perplexed by the decision to admit this patient to General Medicine, since patients undergoing active chemotherapy are normally admitted to the Oncology service. The information provided in the text of the page gives rise to a question in his mind: "So, why is she coming to us?" A sensemaking process is triggered, directing the physician's information seeking in the patient's record. He looks for some rationale as to why the patient would be admitted to General Medicine. Rather than aimlessly browsing the record, physicians look at particular parts of the record for specific pieces of information, based on how the patient's complaint is framed in the admission page. One hospitalist explained:

If it's a patient with heart failure, I'll look for their last echocardiogram to see what their heart function is like, or someone coming in with chest pain, I'll see if they've ever had a stress test or something like that. (P105)

Chest pain, to take one of the examples from this quote, is a symptom of multiple illnesses, representing a considerable range of severity, which in turn might suggest that different levels of care or types of sub-specialty expertise are needed. The physician looks into the patient's record for particular tests and examines the results to evaluate the severity, form a differential diagnosis, and assess the disposition plan. As a result, he enters the handoff knowing concrete details about the patient's condition and care rather than just knowing that the patient has "chest pain." 


\section{Questions Arise from the Chart Biopsy}

Concerns not only direct the information seeking during chart biopsy, but they also emerge during the process. While reviewing the patient's record, General Medicine physicians sometimes developed questions and concerns based on the information they encountered in the record. That is, the process of making sense is not only an effort to span gaps in one's understanding, but may also uncover new gaps as well. The following field note excerpt demonstrates how this happens.

P107 looks up the patient's record in the system. "Nothing scary yet," she says while scanning the previous outpatient and inpatient visit records. P107 realizes that the patient just left Ortho [orthopedic surgery] two days before. "Why is this one coming in and why to us?" She reads on, then remarks out loud, "Why's she coming in? And Ortho won't take their own patient?" She turns to another doctor in the room and says, "She's coming in for pain, and she had surgery three days ago. Oh, how irritating!" (P107)

This chart biopsy began as an effort to gain a quick overview of the patient, but concerns about the disposition plan emerged and the physician's affective state changed once she encountered information about a recent hospitalization for surgery. The normal practice at the study site is that patients being readmitted within seven days of a discharge should be admitted to the same service that previously cared for them unless the new admission is completely unrelated. As a result of the chart biopsy, this hospitalist's understanding of the patient went from "nothing scary" to the conclusion that the patient belonged on a surgical service, and she entered the handoff irritated with the disposition plan.

The questions that emerge during the chart biopsy are not only those which assess the disposition plan, but also those which address the ongoing care of the patient. One resident offered an example.

So if you can look the patient up before you have that conversation you already know five questions you're going to ask them [the ED physician] because you see, "Oh, they had a renal transplant," and "How's that doing? Did you guys send these tests?" Because there's certain tests, you know, they might come in with a complaint of a headache, you know, or something and have a new brain tumor, but if they have a renal transplant you always need to check and see how that's doing and check the levels of their antirejection drugs they're taking et cetera. So, you know, as a side point: "Did you send those tests off? Did you think to see how their status is?" I find that that is more helpful if I can look the patient up before I call them. (P110)

Information uncovered during the chart biopsy can trigger the receiving physician to ask pointed questions during the handoff, potentially ensuring that important aspects of the patient's care are not overlooked during the transition of responsibility and control.

\section{Variations in Practice}

The practice of pre-handoff chart biopsy can vary, at times considerably, from one physician to the next and even from one instantiation to the next by the same physician. Although no attempt was made to time all chart biopsies, some were observed to last less than thirty seconds, while others lasted more than ten minutes. Some chart biopsies involved accessing both EDCentral and MedRec. Others involved accessing only one system. Two physicians in particular, each observed on multiple occasions, were regularly methodical in their chart biopsies and appeared to spend more time and explore more aspects of the record than did many of their peers. Other physicians displayed more irregularity in performing chart biopsies. These sometimes scanned records quickly and briefly, while other times they read portions more carefully. At times they sought the answer to a single question, while other times they looked for information relevant to multiple concerns. Many of the physicians who participated were observed, at some point during the study, to take a handoff without performing any semblance of a chart biopsy beforehand.

Variations in practice of chart biopsy may be partially understood as responses to variations in patients and the way patients are presented in the text of admission pages. Some patients are more complex, with various comorbidities and complicated past medical histories that give rise to multiple concerns about appropriate disposition and treatment, for instance, while other patients' cases are more straightforward and clear protocols exist to guide disposition and care. The concerns that arise regarding the former group of patients can stimulate a desire to explore more of the record than is often the case with the latter group. Practice variations may also be understood as responses to situational demands, specifically time constraints and other concerns competing for the physician's attention, as one hospitalist explains:

Well by now I do have a system, unless, let's say, I'm swamped and I'm in seeing a patient and ER keeps paging me, paging me, paging me. I step out and just call without even looking up. I take the story, finish what I'm doing, and then go back and look up and if there are any issues then I call them again. (P112)

Physicians may spend less time and conduct less thorough chart biopsies or not conduct a pre-handoff chart biopsy at all, as the quote shows, because of situational constraints. At other times no pre-handoff chart biopsy is performed because the ED physician does not include sufficient details, namely the patient's ID number, in the text of the admission page so that the General Medicine physician can locate the correct record. In other cases, the patient is new to the system, with no previous records on file and only a minimal record of the ED's activities in EDCentral is available, limiting the General Medicine physician's ability 
to construct a detailed understanding of the patient. Recognizing that the practice of performing a pre-handoff chart biopsy is a variable one leads to the realization that General Medicine physicians enter different admission handoffs with different degrees of understanding of the patient. As a result, the interaction during handoff may be expected to vary.

\section{Consequences for Handoff}

Physicians in both the ED and General Medicine services suggested that chart biopsies impact the content and nature of handoff interactions.

I think from my end [when I don't look up the patient's record before taking the handoff] it's just a lot more at the mercy of the emergency room residents, what they're telling me, what the story is, and I just say, "Okay," and I don't usually have-I may have a few questions that came up from what they're telling me, but, you know, I don't really have any specific questions. Whereas if I would have looked them up before sometimes I will [have specific questions]. (P111)

This resident's comments suggest her own agency both in constructing an understanding of the patient and in interacting during the handoff are affected by the information she gathers (or does not gather) prior to taking the handoff. Without a pre-handoff chart biopsy, she is "at the mercy of the emergency room resident." In other words, a pre-handoff chart biopsy may empower the receiving physician to take a more active role in the handoff. General Medicine physicians perceived that they asked better questions during handoff and that the use of time during handoff was more efficient as a result of the pre-handoff chart biopsy. Reducing unnecessary admissions and inappropriate placements were also cited as benefits of prehandoff chart biopsy.

\section{DISCUSSION}

The pre-handoff chart biopsy is a physician information practice enabled by the advent of the EHR. It is a sensemaking process in which understandings are socially constructed through interactions with the patient record with potential consequences for patient outcomes and the negotiations that constitute the social order of the hospital. Three contributions of this study are discussed below.

First, in describing the pre-handoff chart biopsy, this research provides an account of an information practice not previously documented and characterizes that practice as a social constructionist activity. The information seeking during a chart biopsy is a sensemaking process (Weick, 1995). Physicians move forward in the practice in an attempt to make sense of the patient and the disposition plan. As physicians scan or read the patient record, they are not passively consuming objective information. Rather they are actively assembling an understanding of the experiences and actions of other humans. They are searching the record for indication of rationale, attempting to glean from the documented actions and narratives of their colleagues the intentions and thinking of those colleagues and to consider alternative interpretations of data. In doing so, they make extensive use of their prior knowledge of their colleagues and of the hospital more generally.

Second, this study demonstrates that new information technologies can enable new information practices which in turn can alter the balance of participation in organizational coordination efforts. With paper-based records systems, the documents that contain the various pieces of information that physicians need to construct understandings of patients and care efforts are physically dispersed and inaccessible given time constraints. With such systems, a physician on the receiving end of an admission handoff enters the handoff conversation with little or no information about the patient and largely "at the mercy" of the physician handing off responsibility.

The advent of the EHR changed this by enabling the prehandoff chart biopsy. By scanning a patient's record before taking the handoff, physicians on the receiving end are able to construct their own initial understandings of patients and to use these understandings during the handoff to engage their colleagues more actively, to ask different kinds of questions, often more pointed towards specific aspects of the patient's case. As a result of the pre-handoff chart biopsy, the General Medicine physician may enter the handoff with an understanding of the patient that differs from the understanding held by the ED physician, and these differing interpretations will need to be negotiated during the handoff. Physicians who conduct pre-handoff chart biopsies enter the handoff not as passive recipients of information who are at the mercy of their coworkers, but as active co-constructors of an understanding of the patient. The nature of organizational coordination can therefore be altered as a result of new information practices.

Third, the study shows that the understandings constructed from information seeking can have consequences for organizational orders. Hospitals are complex organizations of diverse yet limited resources (e.g., specialists, diagnostic technologies, beds, etc.) that need to be allocated to a variable and unpredictable influx of patients. In addition to addressing concerns about efficiency, hospitals must organize to provide care that ensures safety and quality as well. Care transitions such as handoffs between hospital units engender potential threats to quality and safety (Behara, et al., 2005). Pre-handoff chart biopsies affect the negotiations surrounding such transitions, influencing the way the hospital makes sense of patients and consequently organizes its resources to address the needs of those patients. Armed with information gleaned from the chart biopsy, physicians may propose alternative understandings of patients and suggest or even demand different approaches to disposition and care. Knowledge gleaned from a chart biopsy may better position the receiving party to act as a fresh set of eyes to catch errors and oversights. 
As a result of information seeking, organizational orders may be changed: workflows may be altered, resources may be utilized differently, and organizational resilience may be improved.

This study demonstrates the usefulness of an information behavior lens for understanding the impacts of new information systems in general, and sheds light on one use of EHRs in particular. The design and evaluation of futuregeneration EHRs should explore how better to support prehandoff chart biopsy and the sensemaking processes involved in constructing understandings of patients. Similarly, in documenting the processes of constructing understandings through chart biopsies and highlighting the perceived impacts of such understandings on handoff interactions, this research demonstrates that characterizing handoff as an information exchange understates the complexity of the activity, at least in some instances. Recasting handoff as a co-construction of understanding, where differing interpretations must be negotiated, has implications for practitioners and policymakers who seek to improve care transitions. For example, handoff protocols and practices that emphasize the active role of the receiver and that facilitate co-constructed understandings may be needed. Similarly, improving the skills of clinicians to perform chart biopsies may have positive consequences for improved safety, quality and efficiency. Finally, this study has implications for organizing and the negotiations that coordinate work across organizational boundaries, such as between hospital services or corporate departments. The availability of information and the process of seeking for and constructing understandings from that information prior to and during coordination efforts can have consequences for the utilization of resources and can impact the organization's ability to catch errors and otherwise organize effectively and safely.

\section{CONCLUSION}

Through examination of one information seeking practice, this study has demonstrated that information behaviors can be characterized as social constructionist processes with consequences for organizational orders. While generalizing from ethnography is problematic, the findings do suggest conceptual framings that may be useful for studying information seeking and use in other institutions and organizational domains. Further research could explore the link between information practices and organizational orders to collectively build theory to explain the relationship between information behaviors and organizational change and stability.

\section{ACKNOWLEDGEMENTS}

The author is deeply grateful to Michael Cohen, Kai Zheng, and three anonymous reviewers for constructive comments on previous drafts and to the clinicians who graciously permitted this study of their work. This project was supported by a University of Michigan Rackham Graduate Student Research Grant, the ProQuest Dissertation Writing
Award, and by grant number R36HS018758 from the Agency for Healthcare Research and Quality. The content is solely the responsibility of the author and does not necessarily represent the official views of the Agency for Healthcare Research and Quality or of other funders. This study was approved by a university institutional review board.

\section{REFERENCES}

Apker, J., Mallak, L. A., \& Gibson, S. C. (2007). Communicating in the "gray zone": perceptions about emergency physician hospitalist handoffs and patient safety. Academic Emergency Medicine, 14(10), 884-894.

Behara, R., Wears, R., Perry, S., Eisenberg, E., Murphy, L., Vanderhoef, M., et al. (2005). A Conceptual Framework for Studying the Safety of Transitions in Emergency Care. Advances in Patient Safety, vol 2. http://www.ahrq.gov/downloads/pub/advances/vol2/Beha ra.pdf.

Brown, P. J., Borowitz, S. M., \& Novicoff, W. (2004). Information exchange in the NICU: what sources of patient data do physicians prefer to use? International Journal of Medical Informatics, 73(4), 349-355.

Charmaz, K. (2006). Constructing Grounded Theory: A Practical Guide Through Qualitative Analysis. Thousand Oaks, CA: Sage.

Cohen, M. D., \& Hilligoss, P. B. (2008). Handoffs in Hospitals: A review of the literature on information exchange while transferring patient responsibility or control. http://hdl.handle.net/2027.42/61498.

Cohen, M. D., \& Hilligoss, P. B. (in press). The Published Literature on Handoffs in Hospitals: Deficiencies Identified in an Extensive Review. Quality \& Safety in Health Care.

Covell, D., Uman, G., \& Manning, P. (1985). Information needs in office practice: are they being met? Annals of Internal Medicine, 103, 596-599.

Dawes, M., \& Sampson, U. (2003). Knowledge management in clinical practice: a systematic review of information seeking behavior in physicians. International Journal of Medical Informatics, 71(1), 9-15.

Dervin, B. (1999). On studying information seeking methodologically: the implications of connecting metatheory to method. Information Processing \& Management, 35(6), 727

Forsythe, D. E., Buchanan, B. G., Osheroff, J. A., \& Miller, R. A. (1992). Expanding the concept of medical information: An observational study of physicians' information needs. Computers and Biomedical Research, 25(2), 181-200.

Furukawa, M. F., Raghu, T. S., \& Shao, B. B. M. (2010). Electronic Medical Records, Nurse Staffing, and Nurse- 
Sensitive Patient Outcomes: Evidence from California Hospitals, 1998-2007. Health Services Research. http://dx.doi.org/10.1111/j.1475-6773.2010.01110.x

Gorman, P. N., Ash, J., Lavelle, M., Lyman, J., Delcambre, L., Maier, D., et al. (2000). Bundles in the Wild: Managing Information to Solve Problems and Maintain Situation Awareness. Library Trends, 49(2), 266.

Gorman, P. N., \& Helfand, M. (1995). Information Seeking in Primary Care: How Physicians Choose Which Clinical Questions to Pursue and Which to Leave Unanswered. Medical Decision Making, 15(2), 113-119.

Green, M. L., Ciampi, M. A., \& Ellis, P. J. (2000). Residents' medical information needs in clinic: are they being met? The American Journal of Medicine, 109(3), 218-223.

Hartswood, M., Procter, R., Rouncefield, M., \& Slack, R. (2003). Making a Case in Medical Work: Implications forthe Electronic Medical Record. Computer Supported Cooperative Work, 12(3), 241-266.

Hersh, W. R. (1995). The electronic medical record: Promises and problems. Journal of the American Society for Information Science, 46(10), 772-776.

Hillestad, R., Bigelow, J., Bower, A., Girosi, F., Meili, R., Scoville, R., et al. (2005). Can Electronic Medical Record Systems Transform Health Care? Potential Health Benefits, Savings, And Costs. Health Affairs, 24(5), 1103-1117.

Jones, D., Bates, S., Warrillow, S., Opdam, H., Goldsmith, D., Gutteridge, G., et al. (2005). Circadian pattern of activation of the medical emergency team in a teaching hospital. Critical Care, 9(4), R303-306.

Nugus, P., Bridges, J., \& Braithwaite, J. (2009). Selling patients. BMJ (Clinical Research Ed.), 339(19-26 Dec), 1444-1446.

Osheroff, J. A., Forsythe, D., Buchanon, B., Bankowitz, R., Blumenfeld, B., \& Miller, R. (1991). Physicians' information needs: analysis of questions posed during clinical teaching. Annals of Internal Medicine, 114(7), 576-581.

Patterson, E. S., Roth, E. M., Woods, D., D., Chow, R., \& Gomes, J. O. (2004). Handoff strategies in settings with high consequences for failure: Lessons for health care operations. International Journal for Quality in Health Care, 16(2), 125-132.

Petersen, L., Brennan, T., O'Neil, A., Cook, E., \& Lee, T. (1994). Does Housestaff Discontinuity of Care Increase the Risk for Preventable Adverse Events? Annals of Internal Medicine, 121(11), 866-872.

Reddy, M. C., \& Dourish, P. (2002). A finger on the pulse: Temporal rhythms and information seeking in medical work. In Proceedings of the 2002 ACM conference on computer supported cooperative work. ACM.

Reddy, M. C., Pratt, W., \& Dourish, P. (2002). Asking Questions: Information Needs in a Surgical Intensive Care Unit. In, Proceedings of the 2002 AMIA Annual Symposium. AMIA.

Saldana, J. (2009). The Coding Manual for Qualitative Researchers. London: SAGE.

Savage, R. (1996). What clinical information do doctors need? (Information in Practice, part 1). British Medical Journal, 313(7064), 1062-1067.

Sidorov, J. (2006). It Ain't Necessarily So: The Electronic Health Record And The Unlikely Prospect Of Reducing Health Care Costs. Health Affairs, 25(4), 1079-1085.

Strauss, A. (1978). Negotiations. San Francisco, CA: Jossey-Bass Inc.

Strauss, A., Schatzman, L., Ehrlich, D., Bucher, R., \& Sabshin, M. (1963). The hospital and its negotiated order. In E. Freidson (Ed.), The Hospital in Modern Society (pp. 147-169). New York: Free Press.

Talja, S. (1997). Constituting "information" and "user" as research objects: A theory of knowledge formations as an alternative to the information man-theory. In, Proceedings of an international conference on information seeking in context.

Talja, S., Tuominen, K., \& Savolainen, R. (2005). "Isms" in information science: constructivism, collectivism and constructionism. Journal of Documentation, 61(1), 79.

Tang, P. C., Fafchamps, D., \& Shortliffe, E. H. (1994). Traditional medical records as a source of clinical data in the outpatient setting. In, Proceedings of the 18th Annual Symposium on Computer Applications in Medical Care. Washington: AMIA.

Wears, R. L., Perry, S. J., Stephanie, W., Julia, G., \& James, F. (2007). Emergency department status boards: user-evolved artefacts for inter-and intra-group coordination. Cognition, Technology \& Work, 9, 163170.

Weick, K. E. (1995). Sensemaking in Organizations. Thousand Oaks, CA: Sage Publication. 